\title{
Angelicin inhibits liver cancer growth in vitro and in vivo
}

\author{
FENGLIANG WANG, JUN LI, RONG LI, GUOHUA PAN, MINGXIA BAI and QIANG HUANG \\ Department of Hepatobiliary Surgery, Affiliated Minda Hospital of Hubei Institute \\ for Nationalities, Enshi, Hubei 445000, P.R. China
}

Received March 9, 2016; Accepted June 26, 2017

DOI: $10.3892 / \mathrm{mmr} .2017 .7219$

\begin{abstract}
Previous studies have reported that angelicin exerted antiproliferative effects on several types of tumor cell. However, to the best of our knowledge, the effects of angelicin monotherapy on human liver cancer remain to be investigated. In the present study, the antitumor activity of angelicin was evaluated in vitro and in vivo, and the molecular mechanisms underlying its effects were investigated. The present results revealed that angelicin induced apoptosis in liver cancer cells in a dose- and time-dependent manner. Furthermore, in HepG2 and Huh-7 cells, angelicin-induced apoptosis was demonstrated to be mitochondria dependent, involving the phosphatidylinositol-4,5-bisphosphate 3-kinase/R AC- $\alpha$ serine/threonine-protein kinase signaling pathway. In addition, administration of angelicin to mice bearing liver tumor xenografts inhibited tumor growth, without producing significant secondary adverse effects. These results suggested that angelicin may have potential as a novel therapeutic agent for the treatment of patients with liver cancer.
\end{abstract}

\section{Introduction}

Primary hepatic cancer was reported as the second leading cause of cancer-associated mortality worldwide (1). Approximately $75 \%$ of primary liver cancer cases can be histologically classified as hepatocellular carcinoma (HCC), which causes $>600,000$ cases of mortality each year $(2,3)$. The majority of patients with liver cancer exhibit locally advanced (40-50\%) or metastatic (30-45\%) disease at the time of diagnosis (4), whereas the average 5-year survival rate remains $<37 \%$, despite recent advances regarding multimodal therapeutic strategies (5). The high mortality rates associated with liver cancer have been attributed to a lack of tumor-specific antineoplastic agents (6). Therefore, the identification of novel

Correspondence to: Professor Qiang Huang, Department of Hepatobiliary Surgery, Affiliated Minda Hospital of Hubei Institute for Nationalities, 2 WuFeng Road, Enshi, Hubei 445000, P.R. China E-mail: doctorhuang2008@163.com

Key words: angelicin, hepatocellular carcinoma, apoptosis, mitochondria anticancer agents and therapeutic strategies is imperative for the effective treatment of patients with liver cancer.

Angelicin is among the active compounds isolated from the traditional Chinese herb Angelica archangelica. Angelicin belongs to the chemical class of photosensitizers, which have been used for their antiproliferative activities in various skin diseases $(7,8)$. Angelicin has been reported to exert antiproliferative and genotoxic effects, to induce hemolysis in erythrocytes, and inactivate prokaryotic and eukaryotic microorganisms, and viruses (9-11). In addition, angelicin has been reported to inhibit cell growth and/or induce cell death in several types of tumor $(12,13)$, whereas it demonstrated reduced toxicity in healthy cells (14). However, the efficacy of angelicin against liver cancer, as well as its safety, remain to be elucidated.

The present study evaluated the cytotoxic effects of angelicin on the HepG2 hepatoblastoma and Huh-7 human HCC cell lines, and investigated the mechanisms underlying its actions in vitro and in vivo. Angelicin was demonstrated to inhibit liver cancer growth through the induction of mitochondria-mediated cellular apoptosis. Treatment with angelicin resulted in the downregulation of the anti-apoptotic protein apoptosis regulator $\mathrm{Bcl}-2(\mathrm{Bcl}-2)$ and the activation of the phosphatidylinositol-4,5-bisphosphate 3-kinase (PI3K)/RAC- $\alpha$ serine/threonine-protein kinase (Akt) signaling pathway, ultimately leading to nuclear DNA breakdown and apoptosis. Furthermore, in a mouse orthotopic xenograft model of liver cancer, angelicin administration was revealed to significantly inhibit tumor growth, without exhibiting marked toxicity. These results suggested that angelicin may have potential as a novel therapeutic agent for the treatment of patients with liver cancer.

\section{Materials and methods}

Reagents. Angelicin, dimethyl sulfoxide (DMSO), DAPI, Cell Counting kit 8 (CCK8) assay kit (06432379001), LY294002 hydrochloride (L9908), and 5-bromo-2-deoxyuridine (BrdU) were purchased from Sigma-Aldrich (Merck KGaA, Darmstadt, Germany). Polyclonal antibodies against PI3K (AF3242), Akt (AF6261), phosphorylated (p)-Akt (AF0016), apoptosis regulator BAX (Bax; AF0083) and Bcl-2 (AF6139) were purchased from Affinity Biologicals, Inc. (Ancaster, ON, Canada); antibodies against caspase-9 (9508T), caspase-3 (9665S), cytochrome $c$ (4272S), Ki-67 (12075) and p-vascular endothelial growth factor receptor (VEGFR) 2 (9698) were 
purchased from Cell Signaling Technology, Inc. (Danvers, MA, USA). Horseradish peroxidase-conjugated anti-GAPDH antibody (10494-1-AP), goat anti-mouse immunoglobulin (Ig) G (10317-1-AP) and goat anti-rabbit IgG (10285-1-AP), were obtained from ProteinTech Group, Inc. (Chicago, IL, USA). Dulbecco's modified Eagle's medium (DMEM), fetal bovine serum (FBS), penicillin and streptomycin were purchased from Gibco (Thermo Fisher Scientific, Inc., Waltham, MA, USA). A protease inhibitor cocktail was obtained from Roche Diagnostics (Basel, Switzerland). The bicinchoninic acid (BCA) protein assay and the enhanced chemiluminescence (ECL) kits were obtained from Pierce (Thermo Fisher Scientific, Inc.).

Cell lines and cell culture. HepG2 hepatoblastoma and Huh-7 human HCC cell lines were obtained from the Cell Bank of Shanghai Institute of Biochemistry and Cell Biology, Chinese Academy of Sciences (Shanghai, China). HepG2 cells are a hepatoblastoma cell line known to have been misidentified as an HCC cell line (15). Cells were cultured in DMEM, supplemented with $10 \% \mathrm{FBS}$, and were maintained at $37^{\circ} \mathrm{C}$ in a $5 \% \mathrm{CO}_{2}$ atmosphere.

All experiments were approved by the Hubei University for Nationalities Committee and the Affiliated Minda Hospital Ethics Committee (Enshi, China).

Cellular viability assay. Cellular viability was determined using a Cell Counting kit 8 (CCK8) assay kit, according to the manufacturer's protocol. Cells were seeded into 96-well plates, at a density of $5 \times 10^{3}$ cells/well, and cultured overnight. Various concentrations $(0,2.5,5,10,20,40,60,80,100,120$, 160 and $200 \mu \mathrm{M})$ of angelicin were added to each well for $48 \mathrm{~h}$. Subsequently, CCK8 solution (10 $\mu \mathrm{l})$ was added to each well and cells were incubated at $37^{\circ} \mathrm{C}$ for $3 \mathrm{~h}$. Cell viability was also measured at 12, 24, 36, 48 and $72 \mathrm{~h}$ following treatment with angelicin $(60 \mu \mathrm{M})$. The optical density (OD) of the formed crystals was measured using a 96-well microtiter plate reader (Molecular Devices, LLC, Sunnyvale, CA, USA) to determine the absorbance of each sample at $450 \mathrm{~nm}$. The mean concentration in each set of 3 wells was measured. Viability of the control cells, treated with $0.5 \%$ DMSO, was set as $100 \%$. Percentage viability was calculated using the following formula: \% Viability=(OD of treated cells/OD of control cells) $\mathrm{x} 100$. The CCK8 assay was performed in triplicate.

Colony formation assay. Cells were seeded into 6-well plates and cultured overnight, prior to incubation with various concentrations of angelicin for an additional $48 \mathrm{~h}$. Cells were then maintained for 10 days, with medium being replaced periodically. The resulting colonies were fixed with methanol at $-20^{\circ} \mathrm{C}$ for $30 \mathrm{~min}$ and were stained with $1 \%$ crystal violet for $15 \mathrm{~min}$ at room temperature. Colonies with a diameter $>0.5 \mathrm{~mm}$ were counted under an inverted phase-contrast microscope (Olympus Corporation, Tokyo, Japan).

Flow cytometric analysis. Apoptosis was assessed using the Annexin V-fluorescein isothiocyanate (FITC)/propidium iodide (PI) apoptosis kit (BioVision, Inc., Milpitas, CA, USA), according to the manufacturer's protocol. Briefly, $1 \times 10^{5}$ cells were seeded into 6-well plates and cultured overnight, prior to exposure to various concentrations of angelicin for different times (HepG2 cells $48 \mathrm{~h}$ and Huh-7 $36 \mathrm{~h}$ ). HepG2 cells were treated with $200 \mathrm{nM}$ LY294002 in the absence or presence of angelicin for $24 \mathrm{~h}$. Subsequently, cells were collected and incubated with Annexin V-FITC/PI, according to the manufacturer's protocol. Apoptosis was assessed using a Guava ${ }^{\circledR}$ easyCyte Flow Cytometer (EMD Millipore, Billerica, MA, USA), and the data were analyzed using CellQuest Pro software (version 5.1, BD Biosciences, Franklin Lakes, NJ, USA).

Western blot analysis. Following treatment with different concentrations of angelicin (HepG2 cells with 0, 30, 60 and $90 \mu \mathrm{M}$; Huh-7 cells with for $0,10,30$ and $60 \mu \mathrm{M})$, cells $\left(1 \times 10^{5}\right)$ were collected and lysed in sterile Eppendorf tube, containing $0.1 \mathrm{M}$ phenylmethylsulfonyl fluoride, and a protease and phosphatase inhibitor cocktail, for $30 \mathrm{~min}$ on ice. Lysates were centrifuged at $7,200 \mathrm{xg}$ at $4^{\circ} \mathrm{C}$ for $15 \mathrm{~min}$ and the supernatants were collected. Protein concentration was measured using the BCA method. Equal amounts of extracted protein samples (40 $\mu \mathrm{g} /$ lane) were separated by $12 \%$ SDS-PAGE and transferred onto polyvinylidene difluoride membranes. Membranes were blocked with $5 \%$ bovine serum albumin (BSA; Beyotime Biotechnology, Wuhan, China) for $1 \mathrm{~h}$ at room temperature, probed with primary antibodies (caspase-9, 1:1,000; caspase-3, 1:1,000; cytochrome $c$, 1:2,000; Bax, 1:2,000; Bcl-2, 1:1,000; PI3K, 1:1,000; Akt, 1:2,000 and phosphorylated (p)-Akt, $1: 2,000$ ) overnight at $0-4^{\circ} \mathrm{C}$ in a refrigerator, and subsequently with an appropriate secondary antibody (goat anti-mouse immunoglobulin (Ig) G, 1:4,000; and goat anti-rabbit IgG, 1:4,000) for $1 \mathrm{~h}$ at $4^{\circ} \mathrm{C}$. The protein bands were visualized using an ECL kit and images were captured using an ImageQuant LAS 4000 Mini system (GE Healthcare Life Sciences, Chalfont, UK). The results were quantified by densitometry and processed with the ImageJ software (version 1.46r; National Institutes of Health, Bethesda, MD, USA).

Terminal deoxynucleotidyl transferase dUTP nick-end labeling (TUNEL) assay. For the detection of apoptosis, TUNEL assay was performed, according to the manufacturer's protocol. Cells $\left(1 \times 10^{5}\right)$ were cultured on coverslips and after $24 \mathrm{~h}$, treated with angelicin for different time periods (HepG2 cells $48 \mathrm{~h}$ and Huh-7 $36 \mathrm{~h}$ ). The cells were harvested and fixed with $4 \%$ paraformaldehyde in PBS for $45 \mathrm{~min}$ at room temperature, then permeabilized with $0.25 \%$ Triton X-100 in PBS for $20 \mathrm{~min}$ at room temperature. Subsequently, TUNEL assay was performed according to the manufacturer's protocol and nuclei were stained for $6 \mathrm{~min}$ at room temperature with DAPI. Stained cells were observed under an Axio Imager A1 fluorescence microscope (Zeiss AG, Oberkochen, Germany).

Tumor xenografts in nude mice. Male BALB/c-nu/nu mice (age, 4-6 weeks; mean weight $25 \mathrm{~g} ; \mathrm{n}=18$ ) were obtained from the Animal Experiment Center of Wuhan University of Medicine (Wuhan, China). They were housed at a constant room temperature and humidity with normal air $\left(21 \% \mathrm{O}_{2}\right)$ under a 12-h light/dark cycle with regular diet at room air, they were kept in 4 cages and had access to standard chow and water ad libitum daily. HepG2 cells $\left(1 \times 10^{7}\right)$ were implanted into nude mice via subcutaneous injection in the back and 
A

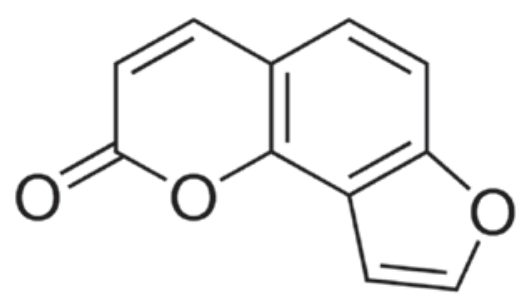

Chemical structure

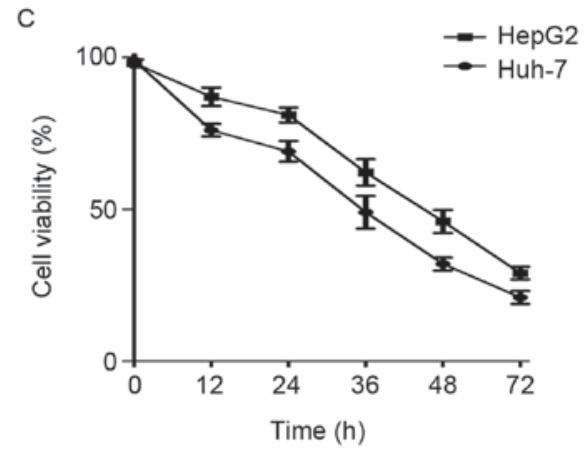

B

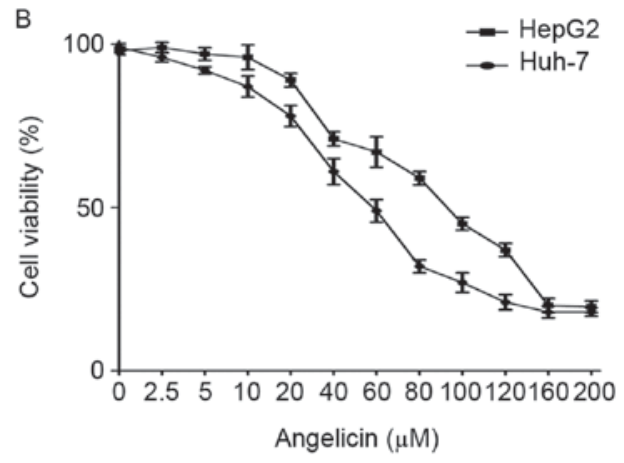

D

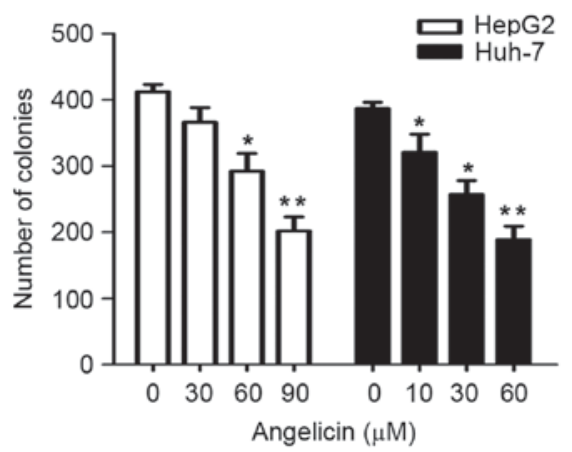

Figure 1. Treatment with angelicin reduces the viability of HepG2 and Huh-7 cells in vitro. (A) Chemical structure of angelicin. (B) HepG2 and Huh-7 cell viability following treatment with various concentrations of angelicin for $48 \mathrm{~h}$, assessed using a CCK8 assay. (C) HepG2 and Huh-7 cell viability following treatment with angelicin for 12, 24, 48 and $72 \mathrm{~h}$, assessed using a CCK8 assay. (D) HepG2 and Huh-7 cell clonogenicity following treatment with various concentrations of angelicin, assessed using a colony formation assay. ${ }^{*} \mathrm{P}<0.05,{ }^{* *} \mathrm{P}<0.01$ vs. the control group $(0 \mu \mathrm{M}$ angelicin). CCK8, Cell Counting kit- 8 .

allowed to grow for $\sim 14$ days to reach a tumor volume of $\sim 100 \mathrm{~mm}^{3}$. Mice were divided into 3 groups ( $\mathrm{n}=6$ mice/group). Mice in the two treatment groups were treated with angelicin (dissolved in saline), at a concentration of 20 or $50 \mathrm{mg} / \mathrm{kg}$ daily, delivered via intraperitoneal injection; mice in the negative control group were treated with an equal volume of vehicle (saline). Tumor growth was monitored by measuring the tumor size every 2 days with a digital caliper. Body weight was monitored every 2 days throughout the treatment period. Mice were sacrificed at the end of the experiment, and tumor xenografts were removed and weighed.

Immunohistochemical staining. Liver tissues from the tumor xenografts or normal controls were removed and fixed in $4 \%$ formalin for $24 \mathrm{~h}$ at $4^{\circ} \mathrm{C}$. Following dehydration, they were embedded in paraffin wax and sectioned at $4 \mu \mathrm{m}$. Sections were deparaffinized in xylene and rehydrated in graded alcohols then treated with a citrate buffer $(\mathrm{pH}=8.0)$ for antigen retrieval in a pressure cooker for $5 \mathrm{~min}$. Subsequently, samples were blocked with $1 \% \mathrm{BSA}$, incubated with a primary antibody Ki-67 (1:100) and p-VEGFR2 (1:50) in a moist chamber overnight at $4^{\circ} \mathrm{C}$, and with a secondary antibody (Histostain-Plus kit; Beyotime Institute of Biotechnology, Wuhan, China) for $30 \mathrm{~min}$ at $37^{\circ} \mathrm{C}$. The values of TUNEL were determined using Apop Tag peroxidase in situ apoptosis detection kits (Merck KGaA, Darmstadt, Germany) in accordance with the manufacturer's protocols and the values of Ki-67 and p-VEGFR2 were determined using the integrated optical density (IOD) at each visual field (magnification, $\mathrm{x} 400$ ) were calculated using
Image-Pro Plus software version 6.0 (Media Cybernetics, Inc., Rockville, MD, USA).

Statistical analysis. The statistical significance of the difference between groups was assessed by student's t tests and one-way analysis of variance followed by Bonferroni post hoc analysis. Data were expressed as the mean \pm standard deviation. $\mathrm{P}<0.05$ was considered to indicate a statistically significant difference. The analysis was performed using GraphPad Prism software version 5.0 (Graph Pad Software, Inc., La Jolla, CA, USA).

\section{Results}

Angelicin inhibits hepatic cancer cell growth in vitro. The chemical structure of angelicin is presented in Fig. 1A. To assess the anticancer effects of angelicin on the HepG2 and Huh-7 cell lines, cellular viability was evaluated using the CCK8 assay. Angelicin was revealed to inhibit the growth of HepG2 and Huh-7 cells in a concentration- and time-dependent manner, with $\mathrm{IC}_{50}$ values of $90 \pm 6.565$ and $60 \pm 4.256 \mu \mathrm{M}$, respectively, following $48 \mathrm{~h}$ of treatment (Fig. 1B and C). A colony formation assay was used to evaluate the long-term effects of angelicin on cell survival. The present results demonstrated that angelicin inhibited the formation of HepG2 and Huh-7 colonies in a concentration-dependent manner (Fig. 1D). Upon observation, HepG2 and Huh-7 cells treated with increasing concentrations of angelicin appeared to shrink in size and lose their adhesion capabilities (Fig. 2A and B). 

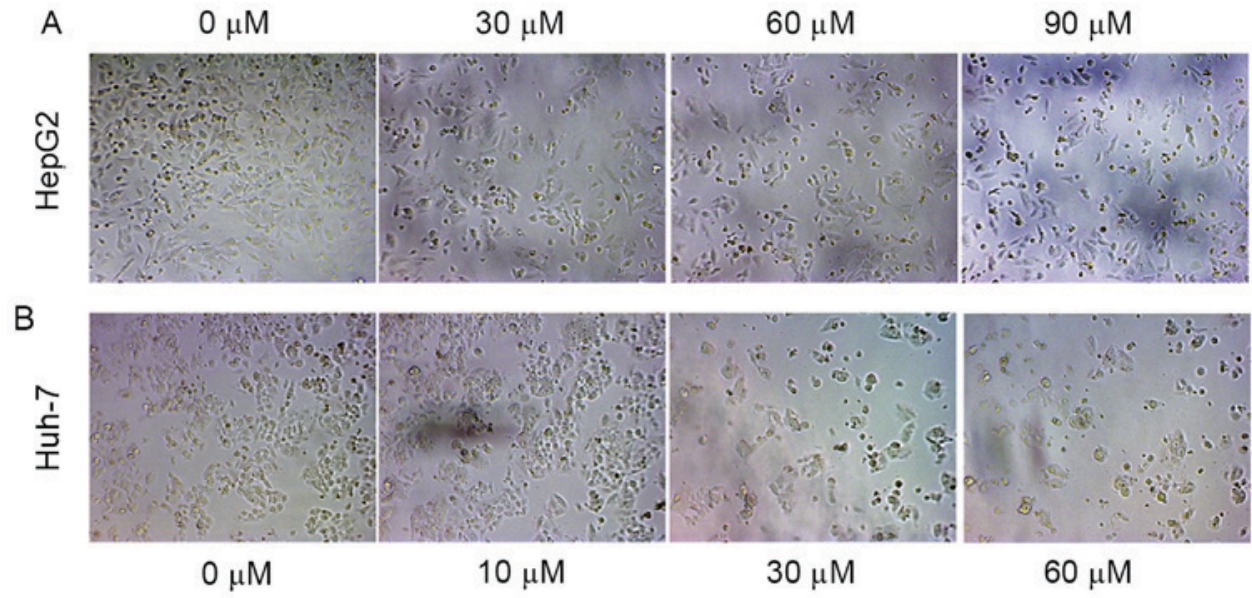

Figure 2. Treatment with angelicin alters the morphology of HepG2 and Huh-7 cells in vitro. (A) HepG2 cellular morphology following treatment with angelicin. (B) Huh-7 cellular morphology following treatment with angelicin. Magnification, x200.
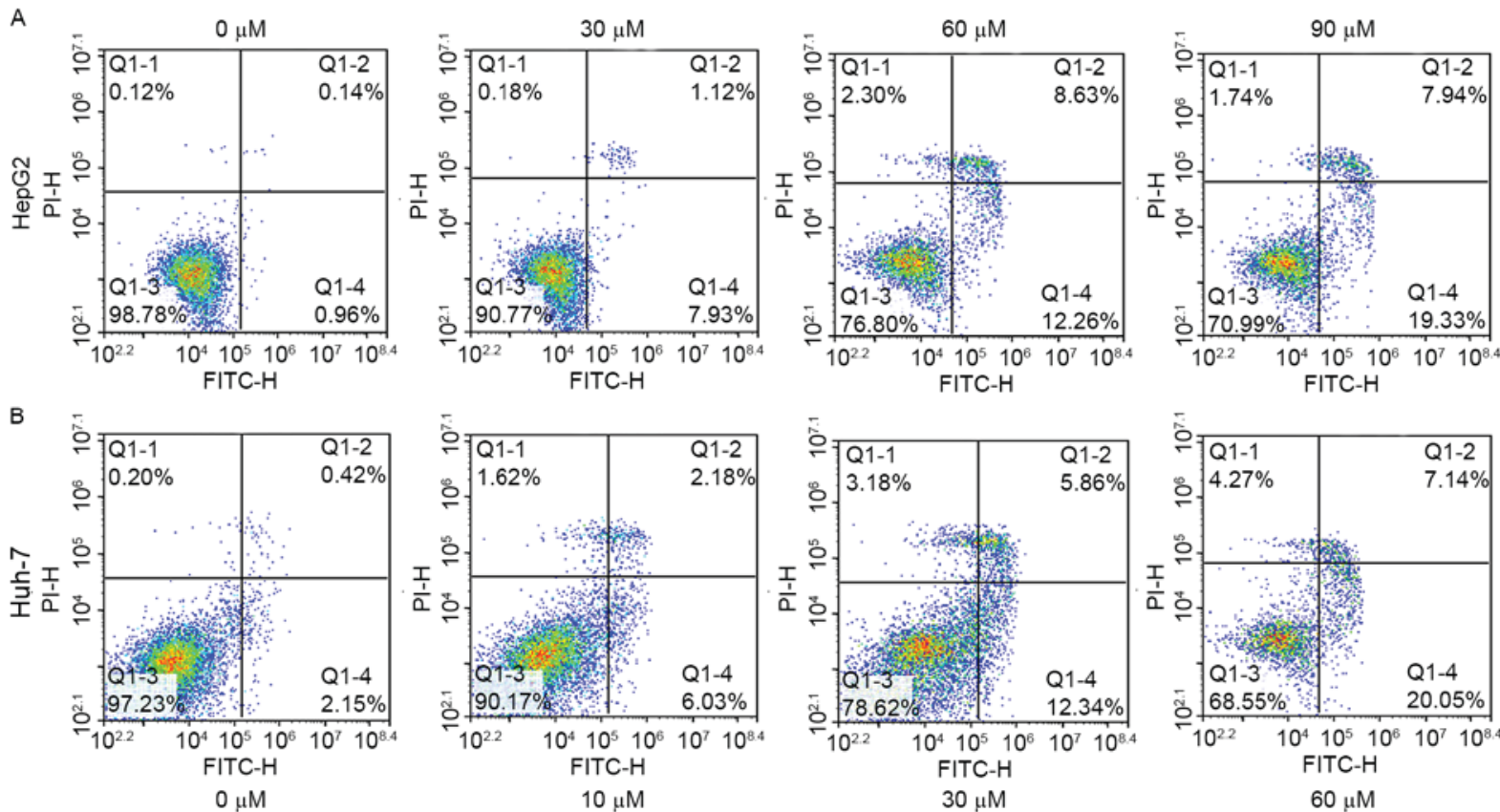

Figure 3. Treatment with angelicin induces apoptosis in HepG2 and Huh-7 cells in vitro. Flow cytometric analysis of (A) HepG2 and (B) Huh-7 cells following treatment with angelicin, using Annexin V-FITC/PI staining. FITC, fluorescein isothiocyanate; PI, propidium iodide; Q, quadrant.

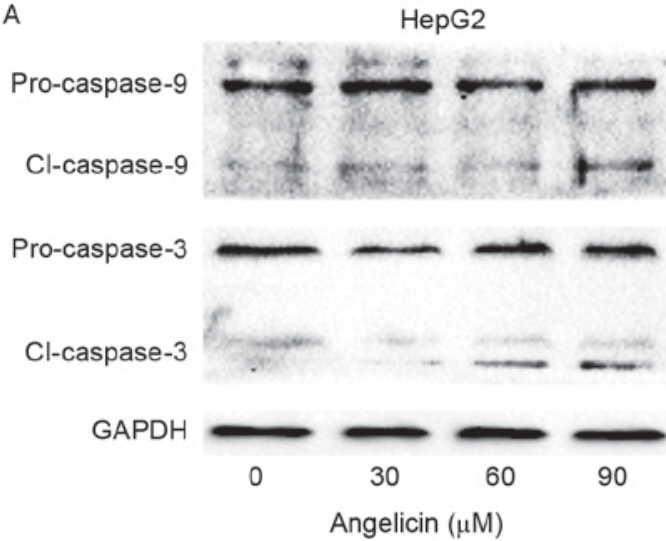

B

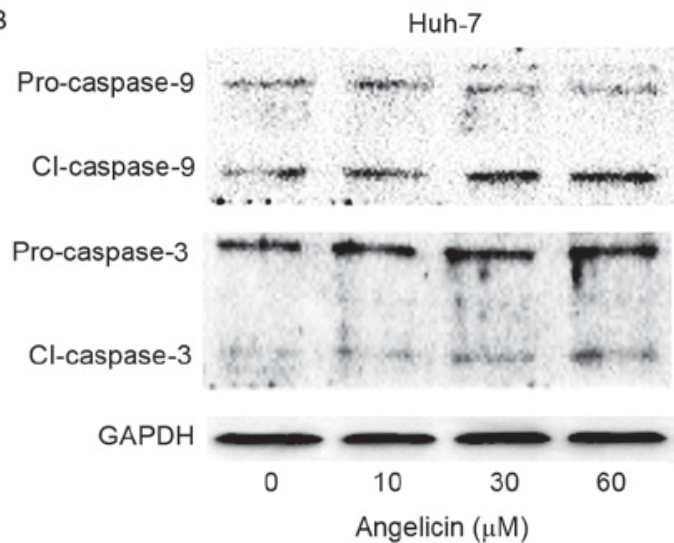

Figure 4. Treatment with angelicin increases caspase- 9 and caspase-3 activation in vitro. Treatment with angelicin activated caspase cleavage in a concentration-dependent manner in (A) HepG2 cells and (B) Huh-7 cells. Western blot analysis was performed using antibodies against proteins associated with caspase-dependent apoptosis. $\mathrm{Cl}$, cleaved. 
A
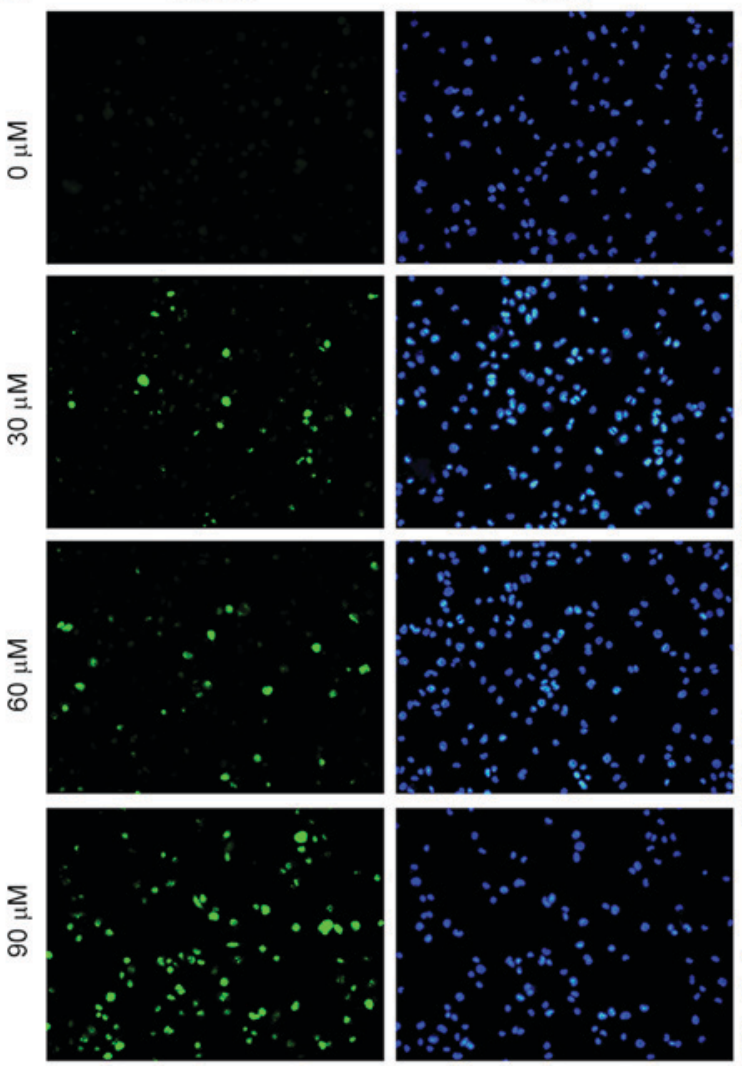
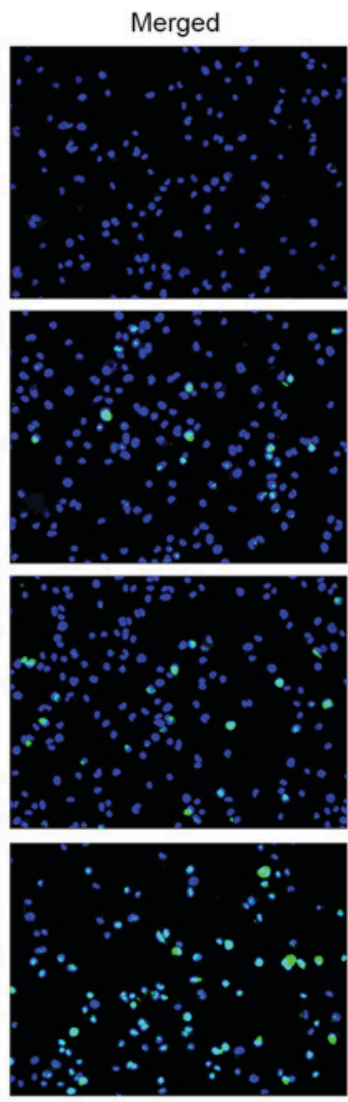

B

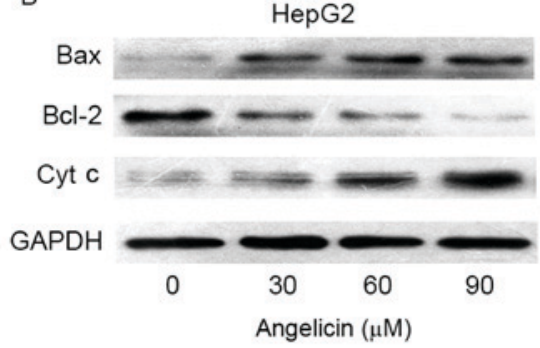

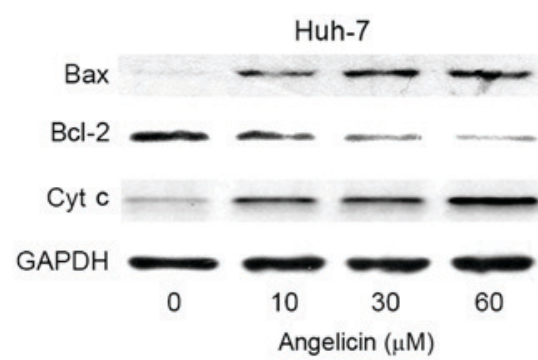

Figure 5. Treatment with angelicin induces apoptosis via a mitochondrial pathway in HepG2 and Huh-7 cells in vitro. (A) Representative photomicrographs demonstrate the results of the TUNEL assay. Alterations in apoptotic cells were assessed using the ratio of green fluorescence. (B) Protein expression levels of Bcl-2, Bax and cytochrome $c$ in HepG2 and Huh-7 cells following treatment with angelicin were assessed using western blot analysis. Bcl-2, apoptosis regulator Bcl-2; Bax, apoptosis regulator BAX; TUNEL, terminal deoxynucleotidyl transferase dUTP nick-end labeling; Cyt c, cytochrome $c$.

These results suggested that angelicin exerted cytotoxic effects in HepG2 and Huh-7 cells.

Angelicin induces cellular apoptosis and increases caspase-9 and caspase-3 activity. To investigate the proapoptotic effects of angelicin, HepG2 and Huh-7 cells were treated with various concentrations of angelicin for $48 \mathrm{~h}$. Apoptosis was assessed using Annexin V-FITC/PI double-staining and flow cytometry. The percentage of apoptotic HepG2 and Huh-7 cells, including early and late apoptotic cells, increased following treatment with angelicin in a concentration-dependent manner (Fig. 3A and B).

The activation of caspases is considered to be a hallmark of apoptosis, and caspase- 9 and caspase- 3 are critical molecules within the caspase apoptotic cascade $(16,17)$. In the present study, western blot analysis was used to assess caspase activation. Cleaved caspase- 9 and cleaved caspase- 3 protein expression appeared to be upregulated in HepG2 and Huh-7 cells following treatment with angelicin (Fig. 4A and B). These results suggested that angelicin may induce apoptosis in HepG2 and Huh-7 cells via enhancing caspase-9 and caspase-3 activity.

Angelicin induces apoptosis through mitochondrial pathways. The proapoptotic effects of angelicin in cancer cells were also assessed using a TUNEL assay. The present results demonstrated that angelicin induced HepG2 and Huh-7 cell apoptosis in a dose-dependent manner (Fig. 5A).

Mitochondria serve important roles in the regulation of cellular apoptosis, through the release of proapoptotic factors (18). Bcl-2 has been demonstrated to inhibit apoptosis via preventing the mitochondrial release of cytochrome $c$ and inhibiting caspase activation (19), whereas Bax has been revealed to induce apoptosis via disintegrating the outer 
A

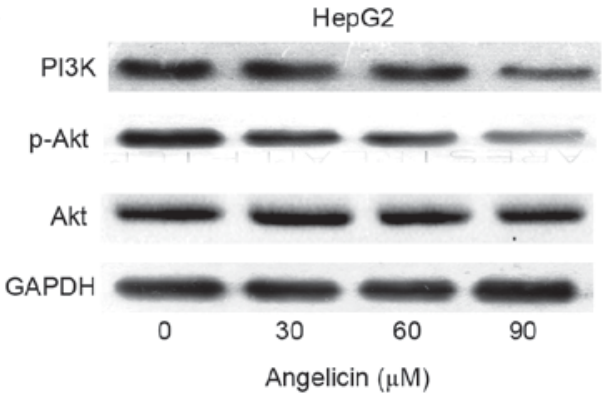

Huh-7

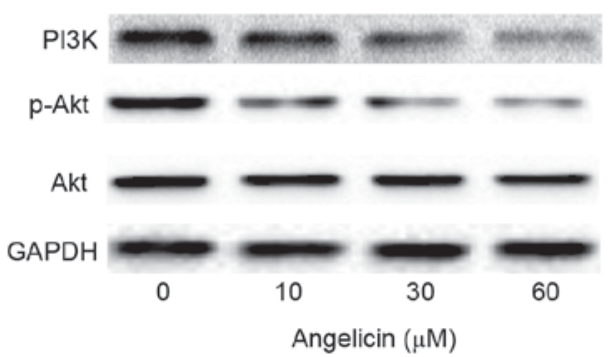

B

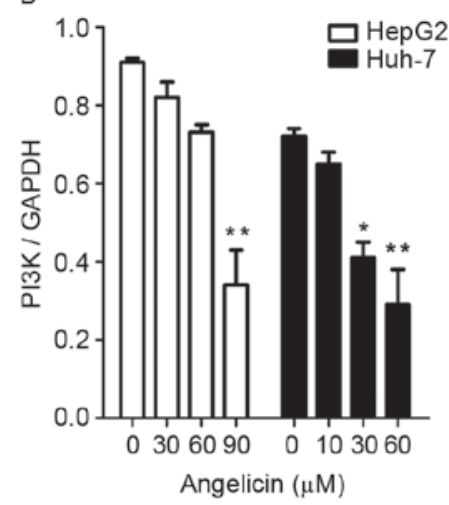

C

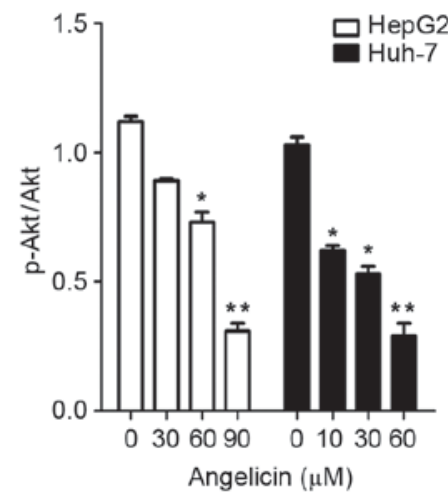

D

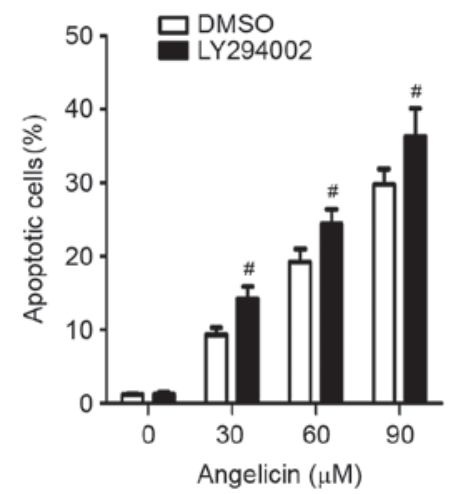

Figure 6. Treatment with angelicin alters the protein expression levels of PI3K, p-Akt and total Akt in HepG2 and Huh-7 cells in vitro. (A) Representative blots demonstrating PI3K, p-Akt and total Akt protein expression levels in HepG2 and Huh-7 cells, following treatment with various concentrations of angelicin. (B) PI3K blots were semi-quantified using densitometry analysis of the protein bands and normalized to GAPDH. (C) p-Akt/Akt blots were semi-quantified using densitometry analysis of the protein bands. (D) Effects of LY294002 on angelicin-induced apoptosis. Cells were treated with the PI3K inhibitor LY294002 (3 mM) for $1 \mathrm{~h}$ prior to treatment with angelicin. The percentage of apoptotic cells following treatment with angelicin in the presence or absence of LY294002 was assessed using Annexin V-fluorescein isothiocyanate/propidium iodide staining and flow cytometry. Data are expressed as the mean \pm standard deviation of 3 independent experiments. ${ }^{*} \mathrm{P}<0.05,{ }^{* *} \mathrm{P}<0.01$ vs. the control group $\left(0 \mu \mathrm{M}\right.$ angelicin); ${ }^{~} \mathrm{P}<0.05$ vs. the DMSO group. PI $3 \mathrm{~K}$, phosphatidylinositol-4,5-bisphosphate 3-kinase; p-, phosphorylated; DMSO, dimethyl sulfoxide; Akt, RAC- $\alpha$ serine/threonine-protein kinase.

A

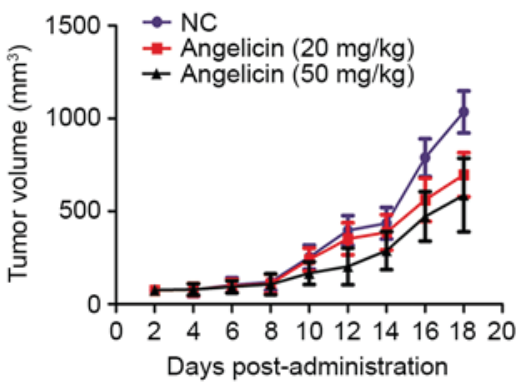

B

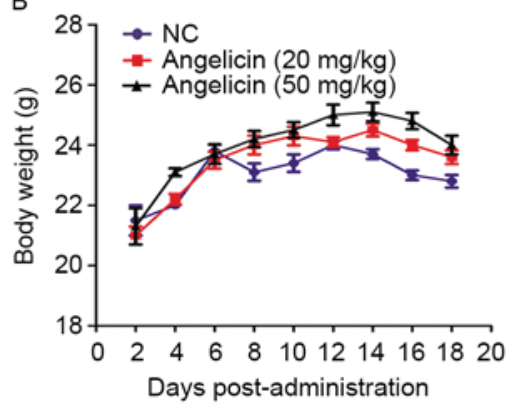

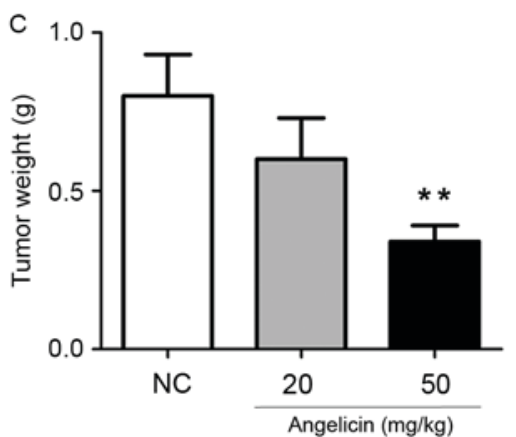

Figure 7. Treatment with angelicin inhibits tumor growth in a mouse orthotopic xenograft model of liver cancer. Following tumor development, mice were randomly assigned into 3 groups ( $\mathrm{n}=6 \mathrm{mice} / \mathrm{group}$ ) and treated daily with $20 \mathrm{or} 50 \mathrm{mg} / \mathrm{kg}$ of angelicin, or with equal volume of saline (NC group), delivered intraperitoneally for 16 days. (A) Tumor volume, (B) body weight and (C) tumor weight were measured. Data are expressed as the mean \pm standard deviation of 6 individual mice in each group from one experiment. ${ }^{* * *} \mathrm{P}<0.01 \mathrm{vs}$. the $\mathrm{NC}$ group. $\mathrm{NC}$, negative control.

mitochondrial membrane and promoting cytochrome $c$ release (20). Therefore, Bcl-2 and Bax expression was investigated in HepG2 and Huh-7 cells following treatment with angelicin. Western blot analysis demonstrated that protein expression levels of Bax were markedly increased, whereas protein expression levels of $\mathrm{Bcl}-2$ were decreased in a dose-dependent manner following angelicin administration, thus leading to an increased $\mathrm{Bax} / \mathrm{Bcl}-2$ ratio (Fig. 5B). Cytochrome $c$ is an apoptotic marker, which, upon release from the mitochondria into the cytosol, can activate caspase- 9 and caspase-3 to execute the apoptotic cascade $(21,22)$. Western blot analysis revealed that angelicin markedly increased cytochrome $c$ protein expression levels. These results suggested that angelicin may induce liver cancer cell apoptosis through the activation of mitochondrial pathways.

Angelicin inhibits PI3K/Akt signaling. The PI3K/Akt signaling pathway is involved in several cellular processes, including survival, proliferation, differentiation and apoptosis (22). The present study examined the effects of angelicin on the 
A
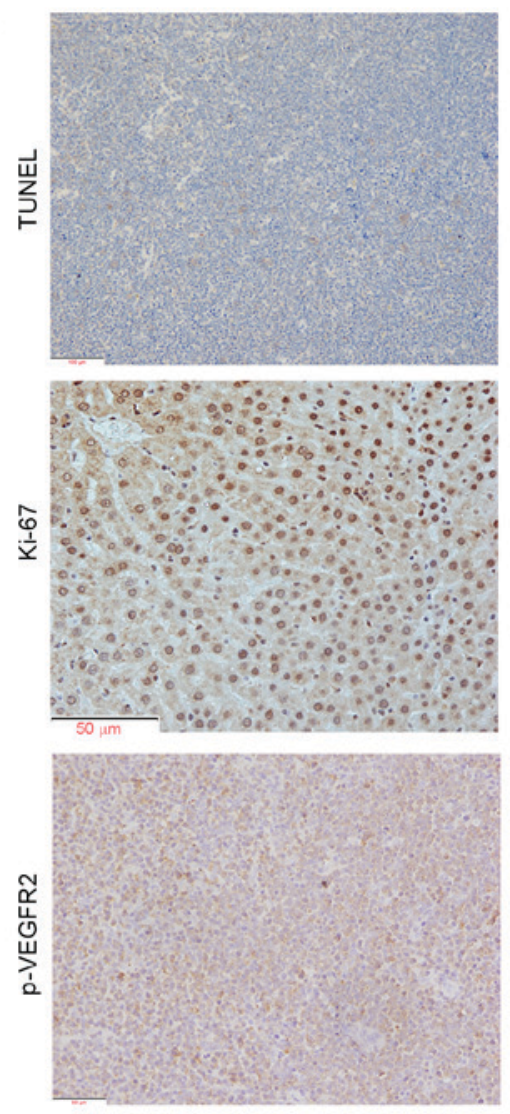

NC

B

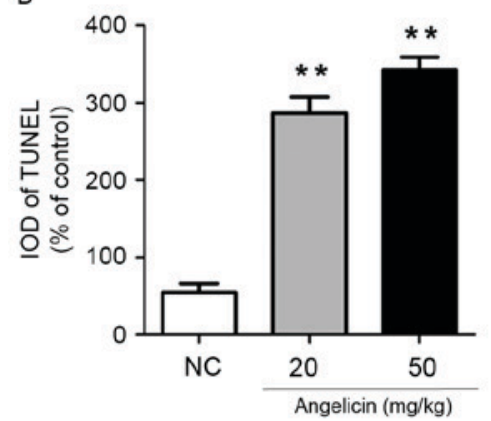

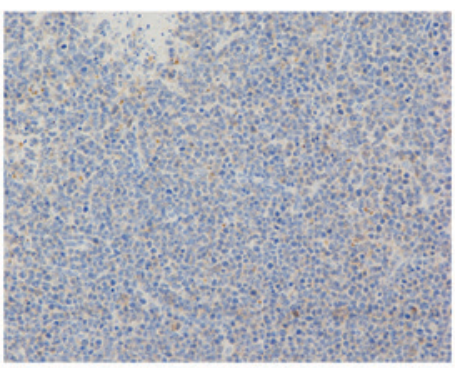
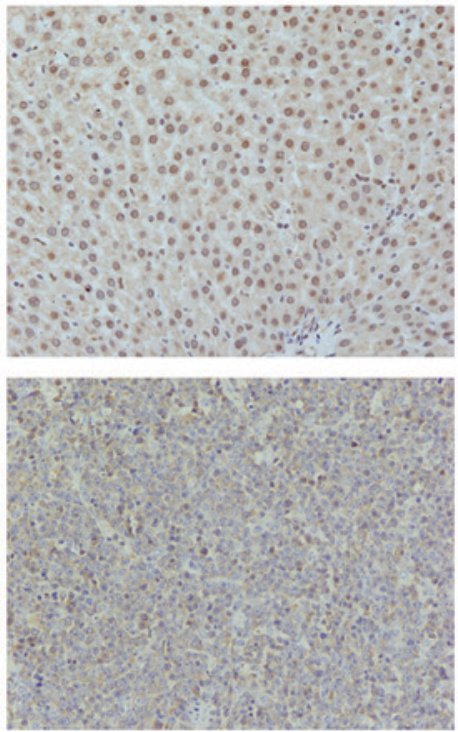

$20 \mathrm{mg} / \mathrm{kg}$

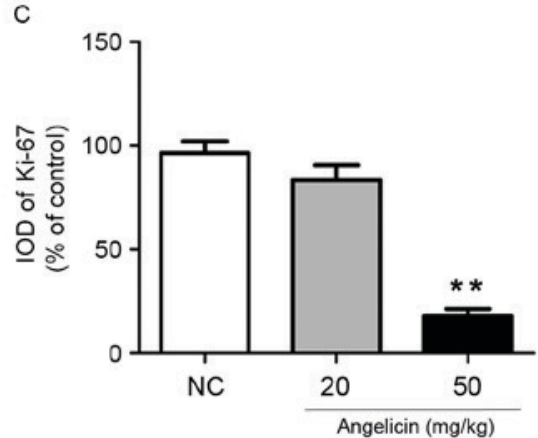

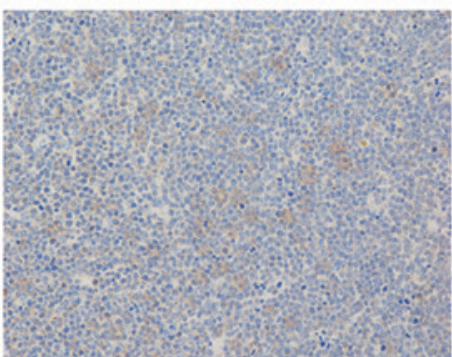
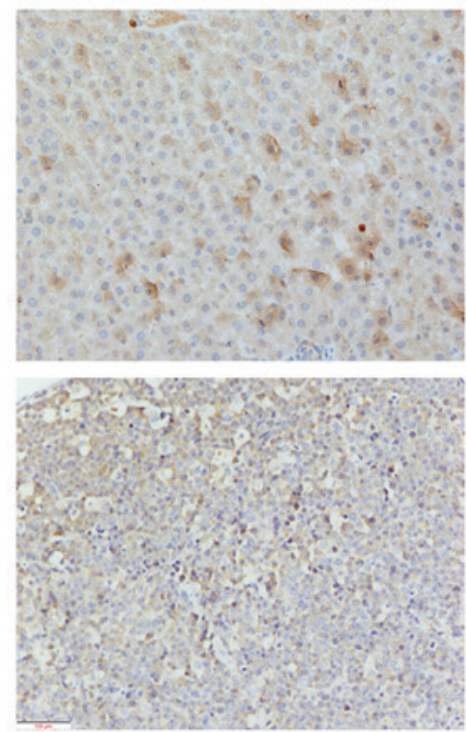

$50 \mathrm{mg} / \mathrm{kg}$

D

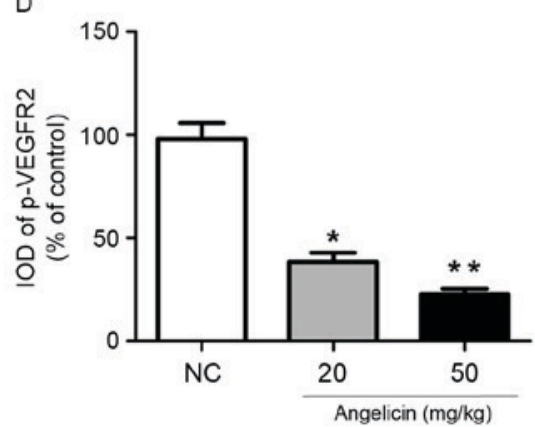

Figure 8. Treatment with angelicin reduced the proliferation and induced the apoptosis of tumor cells, and inhibited angiogenesis in vivo. (A) Representative photomicrographs of immunohistochemical staining for TUNEL, Ki-67 and p-VEGFR2 in tumor tissue samples; magnification, x200. Quantitative results of immunohistochemical staining for (B) TUNEL, (C) Ki-67 and (D) p-VEGFR2. Data are expressed as the mean \pm standard deviation of 6 individual mice in each group from one experiment. ${ }^{*} \mathrm{P}<0.05,{ }^{* *} \mathrm{P}<0.01 \mathrm{vs}$. the NC group. TUNEL, terminal deoxynucleotidyl transferase dUTP nick-end labeling; p-VEGFR2, phosphorylated vascular endothelial growth factor receptor 2; NC, negative control; IOD, integrated optical density.

PI3K/Akt pathway and evaluated whether PI3K/Akt signaling participated in angelicin-mediated apoptotic cell death. Following treatment with angelicin, the protein expression levels of PI3K, Akt and p-Akt were significantly downregulated in a dose-dependent manner (Fig. 6A-C).

To further investigate the contribution of the PI3K/Akt pathway to angelicin-induced apoptosis, the PI3K inhibitor LY294002 was employed. Inactivation of PI3K by LY294002 significantly increased angelicin-induced apoptotic cell death (Fig. 6D). These results suggested that the PI3K/Akt pathway may be implicated in liver cancer cell survival, and its inhibition may be able to potentiate the proapoptotic effects of angelicin.
Angelicin inhibits liver tumor growth in vivo. Based on the aforementioned in vitro results, the effects of angelicin on tumor progression were investigated in vivo, using an orthotopic xenograft model of liver cancer. Notably, treatment with angelicin caused a marked decrease in tumor volume and weight compared with vehicle treatment, whereas it exerted little effect on body weight (Fig. 7A-C). Furthermore, a significant increase in labeling with the apoptotic marker TUNEL and a decrease in the proliferation marker Ki-67 were observed in tumor tissue samples following treatment with angelicin (Fig. 8A). These results suggested that angelicin may suppress tumor growth in vivo, via inhibiting the proliferation and inducing the apoptosis of liver cancer cells. In addition, 
treatment with angelicin resulted in significant reduction of p-VEGFR2 levels in tumor tissue, thus suggesting that disruption of vascularity may participate in the tumor-suppressive effects of angelicin (Fig. 8B-D). No cases of mortality were observed in the angelicin-treated groups. Therefore, it may be hypothesized that angelicin can exert antitumor actions in vivo, possibly via promoting cancer cell apoptosis.

\section{Discussion}

Angelicin is a structural isomer of psoralen and belongs to the furanocoumarin class of chemical compounds, which have been reported to possess anticancer properties $(23,24)$. However, the antitumor effects of angelicin in human liver cancer, as well as the mechanisms underlying its actions, remain to be elucidated. The present study aimed to assess the effects of angelicin on human liver cancer and to investigate the molecular mechanisms underlying its actions. Results revealed that angelicin significantly reduced the viability and promoted the apoptosis of HepG2 and Huh-7 liver cancer cells.

The present study demonstrated that angelicin suppressed the proliferation of HepG2 and Huh-7 cells in a concentration- and time-dependent manner. Mitochondria have been reported to serve essential roles in proapoptotic signaling pathways (25). Angelicin was revealed to induce the activation of caspase-9 and caspase-3 in HepG2 and Huh-7 cells, thus suggesting that mitochondrial processes may be implicated in the proapoptotic actions of angelicin. Members of the Bcl-2 family of proteins serve key roles in the regulation of mitochondrial apoptotic pathways (26). Previous studies have reported that the proapoptotic factor Bax enhanced the mitochondrial release of cytochrome $c$ and activated caspase-9 and caspase- 3 to execute the apoptotic program; conversely, the anti-apoptotic protein $\mathrm{Bcl}-2$ inhibited Bax activation and cytochrome $c$ release, and prevented apoptosis $(27,28)$. Alterations in the $\mathrm{Bax} / \mathrm{Bcl}-2$ ratio have been reported to cause mitochondrial destabilization, which can lead to the release of proapoptotic factors (29). Proapoptotic factors can induce the intrinsic apoptotic pathway by triggering caspase cleavage and activation, and the subsequent initiation of caspase-dependent proteolytic cascades (30). The present results demonstrated that treatment with angelicin resulted in a decrease in Bcl-2 expression and an increase in Bax expression. In addition, protein expression levels of cytochrome $c$ were also decreased following administration of angelicin. These results suggested that mitochondrial processes may be involved in the proapoptotic actions of angelicin.

The PI3K/Akt signaling pathway is involved in numerous cellular processes, including survival, proliferation, differentiation and apoptosis (31). To investigate whether PI3K/Akt signaling may be involved in angelicin-induced apoptosis, HepG2 and Huh-7 cells were treated with various concentrations of angelicin for $48 \mathrm{~h}$. Western blot analysis demonstrated that treatment with angelicin resulted in decreased PI3K expression and Akt phosphorylation compared with vehicle-treated cells. These results suggested that PI3K/Akt signaling may be implicated in angelicin-induced cancer cell apoptosis. To further test the contribution of PI3K/Akt pathways to angelicin-induced apoptosis, cells were treated with LY294002, a specific PI3K inhibitor, in the absence or presence of angelicin for $48 \mathrm{~h}$. The present results revealed that the combination of angelicin and LY294002 exerted a synergistic effect in the induction of apoptosis. These results suggested that PI3K/Akt pathways may be involved in the survival of HepG2 and Huh-7 cells, whereas inhibition of PI3K/Akt signaling may potentiate angelicin-induced apoptosis.

To determine the potential of angelicin to inhibit cancer cell growth in vivo, its antitumor effects were investigated using a liver tumor xenograft model. The present results demonstrated that angelicin inhibited tumor growth and promoted cancer cell apoptosis in vivo. Furthermore, no major toxic adverse events were noted in mice treated with angelicin. Angiogenesis is essential for the establishment of the necessary oxygen and nutrient supply to solid tumors, and thus promotes tumor growth and metastasis (32). To investigate whether the mechanisms underlying the antitumor effects of angelicin involved the inhibition of angiogenetic processes, the expression of p-VEGFR2, a marker of cancer-associated angiogenesis, was assessed using immunohistochemistry. Tumor tissue isolated from mice treated with angelicin exhibited decreased expression of p-VEGFR compared with tissue from untreated control mice. These results suggested that angelicin may suppress tumor growth via disrupting angiogenetic processes and tumor vascularity.

In conclusion, the present study demonstrated that angelicin significantly inhibited the growth of liver cancer cells in vitro and in vivo through the induction of apoptosis. These results suggested that angelicin may have potential as a chemotherapeutic agent against liver cancer cells, acting via inducing cancer cell apoptosis through caspase-dependent mitochondrial pathways. Further studies are required to elucidate the complex molecular mechanisms underlying the effects of angelicin in various types of cancer cells.

\section{References}

1. Trevisani F, Garuti F, Cucchetti A, Lenzi B and Bernardi M: De novo hepatocellular carcinoma of liver allograft: A neglected issue. Cancer Lett 357: 47-54, 2015.

2. Fujita N, Aishima S, Iguchi T, Mano Y, Taketomi A, Shirabe K, Honda H, Tsuneyoshi M and Oda Y: Histologic classification of microscopic portal venous invasion to predict prognosis in hepatocellular carcinoma. Hum Pathol 42: 1531-1538, 2011.

3. Colecchia A, Scaioli E, Montrone L, Vestito A, Di Biase AR, Pieri M, D'Errico-Grigioni A, Bacchi-Reggiani ML, Ravaioli M, Grazi GL and Festi D: Pre-operative liver biopsy in cirrhotic patients with early hepatocellular carcinoma represents a safe and accurate diagnostic tool for tumour grading assessment. J Hepatol 54: 300-305, 2011.

4. Zhu YJ, Zheng B, Wang HY and Chen L: New knowledge of the mechanisms of sorafenib resistance in liver cancer. Acta Pharmacol Sin 38: 614-622, 2017.

5. Lopane C, Agosti P, Gigante I, Sabbà C and Mazzocca A: Implications of the lysophosphatidic acid signaling axis in liver cancer. Biochim Biophys Acta 1868: 277-282, 2017.

6. Li K, Wang HT, He YK and Guo T: New idea for treatment strategies for Barcelona Clinic Liver Cancer stages based on a network meta-analysis. Medicine (Baltimore) 96: e6950, 2017.

7. Kavli G, Midelfart K, Raa J and Volden G: Phototoxicity from furocoumarins (psoralens) of Heracleum laciniatum in a patient with vitiligo. Action spectrum studies on bergapten, pimpinellin, angelicin and sphondin. Contact Dermatitis 9: 364-366, 1983.

8. Lampronti I, Bianchi N, Borgatti M, Fibach E, Prus E and Gambari R: Accumulation of gamma-globin mRNA in human erythroid cells treated with angelicin. Eur J Haematol 71: 189-195, 2003. 
9. Rahman MA, Kim NH, Yang H and Huh SO: Angelicin induces apoptosis through intrinsic caspase-dependent pathway in human SH-SY5Y neuroblastoma cells. Mol Cell Biochem 369: 95-104, 2012.

10. Bordin F, Dall'Acqua F and Guiotto A: Angelicins, angular analogs of psoralens: Chemistry, photochemical, photobiological and phototherapeutic properties. Pharmacol Ther 52: 331-363, 1991.

11. Lampronti I, Bianchi N, Zuccato C, Dall'acqua F, Vedaldi D, Viola G, Potenza R, Chiavilli F, Breveglieri G, Borgatti M, et al: Increase in gamma-globin mRNA content in human erythroid cells treated with angelicin analogs. Int J Hematol 90: 318-327, 2009.

12. Spanò V, Parrino B, Carbone A, Montalbano A, Salvador A, Brun P, Vedaldi D, Diana P, Cirrincione G and Barraja P: Pyrazolo[3,4-h]quinolines promising photosensitizing agents in the treatment of cancer. Eur J Med Chem 102: 334-351, 2015.

13. Seo E, Lee EK, Lee CS, Chun KH, Lee MY and Jun HS: Psoralea corylifolia $\mathrm{L}$. Seed extract ameliorates streptozotocin-induced diabetes in mice by inhibition of oxidative stress. Oxid Med Cell Longev 2014: 897296, 2014.

14. Leonti M, Casu L, Gertsch J, Bonsignore L, Floris C, Casu M and Cottiglia F: A pterocarpan from the seeds of Bituminaria morisiana. J Nat Med 64: 354-357, 2010.

15. López-Terrada D, Cheung SW, Finegold MJ and Knowles BB: HepG2 is a hepatoblastoma-derived cell line. Hum Pathol 40: $1512-1515,2009$

16. Estaquier J, Vallette F, Vayssiere JL and Mignotte B: The mitochondrial pathways of apoptosis. Adv Exp Med Biol 942 $157-183,2012$

17. Platonova A, Koltsova SV, Hamet P, Grygorczyk R and Orlov SN: Swelling rather than shrinkage precedes apoptosis in serum-deprived vascular smooth muscle cells. Apoptosis 17: 429-438, 2012

18. Gillies LA and Kuwana T: Apoptosis regulation at the mitochondrial outer membrane. J Cell Biochem 115: 632-640, 2014.

19. Zeng H, Kong X, Peng H, Chen Y, Cai S, Luo H and Chen P: Apoptosis and $\mathrm{Bcl}-2$ family proteins, taken to chronic obstructive pulmonary disease. Eur Rev Med Pharmacol Sci 16: 711-727, 2012.

20. Zhang Y, Sun S, Chen J, Ren P, Hu Y, Cao Z, Sun H and Ding Y: Oxymatrine induces mitochondria dependent apoptosis in human osteosarcoma MNNG/HOS cells through inhibition of PI3K/Akt pathway. Tumour Biol 35: 1619-1625, 2014

21. Wang X, Chen Y, Wang J, Liu Z and Zhao S: Antitumor activity of a sulfated polysaccharide from Enteromorpha intestinalis targeted against hepatoma through mitochondrial pathway. Tumour Biol 35: 1641-1647, 2014.
22. Singh SS, Yap WN, Arfuso F, Kar S, Wang C, Cai W, Dharmarajan AM, Sethi G and Kumar AP: Targeting the PI3K/Akt signaling pathway in gastric carcinoma: A reality for personalized medicine? World J Gastroenterol 21: 12261-12273, 2015.

23. Hsieh MJ, Chen MK, Yu YY, Sheu GT and Chiou HL: Psoralen reverses docetaxel-induced multidrug resistance in A549/D16 human lung cancer cells lines. Phytomedicine 21: 970-977, 2014.

24. Kleiner HE, Xia X, Sonoda J, Zhang J, Pontius E, Abey J, Evans RM, Moore DD and DiGiovanni J: Effects of naturally occurring coumarins on hepatic drug-metabolizing enzymes in mice. Toxicol Appl Pharmacol 232: 337-350, 2008.

25. Xie J, Xu Y, Huang X, Chen Y, Fu J, Xi M and Wang L: Berberine-induced apoptosis in human breast cancer cells is mediated by reactive oxygen species generation and mitochondrial-related apoptotic pathway. Tumour Biol 36: 1279-1288, 2015.

26. Adem J, Ropponen A, Eeva J, Eray M, Nuutinen U and Pelkonen J: Differential expression of Bcl-2 family proteins determines the sensitivity of human follicular lymphoma cells to dexamethasone-mediated and anti-BCR-mediated apoptosis. J Immunother 39: 8-14, 2016.

27. Wang J, Gao Q, Wang D, Wang Z and Hu C: Metformin inhibits growth of lung adenocarcinoma cells by inducing apoptosis via the mitochondria-mediated pathway. Oncol Lett 10: 1343-1349, 2015.

28. Heinicke U, Kupka J, Fichter I and Fulda S: Critical role of mitochondria-mediated apoptosis for JNJ-26481585-induced antitumor activity in rhabdomyosarcoma. Oncogene 35: 3729-3741, 2016

29. Chen TC, Yu MC, Chien CC, Wu MS, Lee YC and Chen YC: Nilotinib reduced the viability of human ovarian cancer cells via mitochondria-dependent apoptosis, independent of JNK activation. Toxicol in Vitro 31: 1-11, 2016.

30. Zhong F, Yang J, Tong ZT, Chen LL, Fan LL, Wang F, Zha XL and Li J: Guggulsterone inhibits human cholangiocarcinoma Sk-ChA-1 and Mz-ChA-1 cell growth by inducing caspase-dependent apoptosis and downregulation of survivin and Bcl-2 expression. Oncol Lett 10: 1416-1422, 2015.

31. Wang YK, Deng F, Miao J, Xie H and Feng JC: Neuroprotection by Carbenoxolone Against Ischemia Injury Involves PI3K/Akt Pathway. Clin Lab 61: 1561-1568, 2015.

32. Kim DG, Jin Y, Jin J, Yang H, Joo KM, Lee WS, Shim SR, Kim SW, Yoo J, Lee SH, et al: Anticancer activity of TTAC-0001, a fully human anti-vascular endothelial growth factor receptor 2 (VEGFR-2/KDR) monoclonal antibody, is associated with inhibition of tumor angiogenesis. MAbs 7: 1195-1204, 2015. 